\title{
Correspondence
}

\section{EDITED BY KHALIDA ISMAIL}

\section{Contents - Cognitive-behavioural therapy for schizophrenia - Cognitive impairment in bipolar disorder - Antenatal anxiety, parenting and behavioural/emotional problems in children - Brain weight in suicide revisited - Vascular events associated with pharmacotherapy - Outcome measurement in mental health: the Italian experience in psychogeriatrics - Recruitment in old age psychiatry}

\section{Cognitive-behavioural therapy for schizophrenia}

The Insight into Schizophrenia Research Group (Turkington et al, 2002) should be congratulated in reporting results of a large study of cognitive-behavioural therapy (CBT) plus educational handouts $v$. usual care in patients with post-acute schizophrenia. Perhaps because of space restrictions, the article leaves several important questions unanswered which it may be useful to consider here.

Regarding primary outcomes, the authors state that $25 \%$ change would represent 'good clinical improvement' and hence clinical significance but it is notable (from their Table 2: p. 525) that no outcome changed by more than $15 \%$. We assume that the negative change score under burden of care is an error as it does not lie within the standard deviation range. Nevertheless, the authors state that the change in insight was clinically significant in the CBT group with a number needed to treat (NNT) of 10. As it is not stated in the article, perhaps the authors could clarify what thresholds for improvement in the primary outcome measures were chosen in order to calculate the NNTs. Without these, their comparison with the smaller studies by Kuipers et al (1997) and Tarrier et al (1998) (whose main NNTs were both 5 ) is not possible.

Concerning carer participation, it is not clear from the article whether the proportion of patients treated together with a carer was the same in both groups. As satisfaction was rated, how did the $75 \%$ rate of satisfaction in the CBT group compare with that in the treatment-as-usual (TAU) group? This is important because it will affect burden of care ratings and possibly other outcome measures, particularly over the follow-up period.

Perhaps the biggest obstacle in interpreting the study is the question of the equivalence of the arms of the study. This applies not only to the length of time given by the therapists, which should have been controlled, but also the expectation of the patients, and perhaps the educational material given out. Normally, in a randomised controlled trial the patient is blind to the potentially superior intervention. However, in this study 'all TAU patients were told that they would receive CBT intervention at the end of the follow-up period', thus indicating to patients and carers that they were in the control arm of the study.

Despite the large sample size, the study may have still been underpowered to detect the effect of a brief and relatively weak intervention delivered by non-expert therapists. This is best illustrated by re-running the power calculation. Using the authors' own figures, attempting to find an intervention that improves symptomatology by $15 \%$ rather than $25 \%$ would require 1326 participants not 540 . The effects of weak allocation concealment, potentially unequal carer participation and other factors noted by the authors will no doubt impact upon follow-up data but it will be of interest to find out whether the modest effects detected at 5 months will be translated into clinically meaningful changes in readmission rates or functioning at 9 months and beyond (Sederer et al, 1996).

Kuipers, E., Garety, P, Fowler, D., et al (1997) London-East Anglia randomised controlled trial of cognitive-behavioural therapy for psychosis. I: Effects of the treatment phase. British Journal of Psychiatry, I7I, 319-327.

Sederer, L., Dickey, B. \& Hermann, R. (1996) The imperative of outcomes assessment in psychiatry. In Outcomes Assessment in Clinical Practice (eds L. Sederer \& B. Dickey). London: Williams and Wilkins.

Tarrier, N., Yusupoff, L., Kinney, C., et al (1998) Randomised controlled trial of intensive cognitive behaviour therapy for patients with chronic schizophrenia. BMJ, 317, 303-307.

Turkington, D., Kingdon, D., Turner, T., et al (2002) Effectiveness of a brief cognitive-behavioural therapy intervention in the treatment of schizophrenia. British

R. Ruddy, A. Mitchell University of Leeds School of Medicine, Academic Unit of Psychiatry \& Journal of Psychiatry, I80, 523-527.
Behavioural Sciences in Relation to Medicine, 15 Hyde Terrace, Leeds LS2 9LT, UK

Author's reply: We are grateful to Drs Ruddy and Mitchell for the opportunity to clarify the methodology in this study. In relation to the primary outcomes of the study, baseline means are given with change scores and confidence intervals and not standard deviations. The NNT of 10 for a $25 \%$ improvement in insight with CBT was calculated from the following results: 92 out of 257 CBT patients achieved a good clinical improvement compared with 43 out of 165 in the TAU limb. Similarly, a good outcome for overall symptoms was achieved in 112 out of $257 v .59$ out of 165 and for depression in 114 out of $257 v .55$ out of 165 , respectively. The NNTs given can therefore be compared directly with those in the smaller studies listed above. The good outcomes in TAU are interesting and presumably relate to increased use of atypical antipsychotics and the effects of assertive outreach teams. The negative score given for burden of care in the CBT group should indeed be positive, as pointed out in the above correspondence. The proportion of patients treated with a carer was the same in both limbs of the study. However, as TAU patients and carers were not receiving an active comparable intervention to control for therapist time, satisfaction was not rated in that group.

This was the first pragmatic field study designed specifically to answer the question of translation as posed in the Cochrane review (Jones et al, 1999). We were aiming to discover whether any effect would accrue in a community setting with nonexpert therapists using CBT. Having shown a clear effect to be present the study should now be replicated with a control psychological treatment to control for non-specific factors (Sensky et al, 2000). It will be important that this study be run from a position of clinical equipoise as indicated above. The study was adequately powered to detect the symptomatic improvement at the $25 \%$ level as calculated from our pilot study (Turkington \& Kingdon, 2000). The follow-up study will deal with the issue of whether or not these clinically meaningful outcomes are durable and will also include data on relapse.

Jones, C., Cormac, I., Mota, J., et al (1999) Cognitive behaviour therapy for schizophrenia. In Cochrane Collaboration (Issue 4). Oxford: Update Software. 
Sensky, T., Turkington, D., Kingdon, D., et al (2000) A randomized controlled trial of cognitive-behavioural therapy for persistent symptoms in schizophrenia resistant to medication. Archives of General Psychiatry, 57. 165-172.

Turkington, D. \& Kingdon, D. (2000) Cognitivebehavioural techniques for general psychiatrists in the management of patients with psychoses. British Journal of Psychiatry, 177, I0I-106.

\section{Declaration of interest}

The study in question was funded by Pfizer. D.T. has undertaken consultancy work for Pfizer and has received honoraria and hospitality from Pfizer, Janssen and Lilly in relation to conference presentations on the subject of CBT in schizophrenia.

D. Turkington University of Newcastle, School of Neurosciences and Psychiatry, Department of Psychiatry, Royal Victoria Infirmary, Queen Victoria Road, Newcastle upon Tyne NEI 4LP, UK

\section{Cognitive impairment in bipolar disorder}

We write to correct some misconceptions evident in the recent editorial by Ferrier \& Thompson (2002). Previously, we reported impairment in accuracy measures on recognition memory tasks and increased latencies on executive tasks in patients with bipolar disorder in remission (Rubinsztein et al, 2000). Ferrier \& Thompson (2002) argue that the cognitive impairment observed in our study may have been confounded by the effects of 'residual' symptoms. As yet there is no generally accepted 'cut-off' for what constitutes remission. We devised rigorous criteria to define remission based on a patient's own view of his or her illness, that of their psychiatrist and a structured interview. We excluded patients with scores of $\geqslant 8$ on both the Hamilton Rating Scale for Depression (HRSD) and Young Mania Scale (YMS). These rating scales were devised to rate symptom severity in patients with an affective disorder and not for use in normal control subjects. Our average reported score on the HRSD was 2.1 (s.e.m. $=0.5$ ) and on the YMS it was 0.8 (s.e.m. $=0.4$ ). Thus, very few residual symptoms were evident and these scores certainly do not support any concern that patients had residual depression or mania.

Although the rationale for using such scales in controls is dubious, for the sake of argument we have reanalysed our data reported in Rubinsztein et al (2000) using a partial correlation analysis, as in Clark et al (2002), to control for differences observed on the HRSD (we did not rate control subjects using the mania scale) on the tests that showed significant impairment by analysis of variance (ANOVA). We still find significant impairment on both the visual recognition memory tasks and on latency measures from the one-touch Tower of London planning task (see Table 1).

These findings suggest that there are trait impairments in accuracy of visual recognition memory and slower responses on a planning task in bipolar remission. Importantly, impairments of memory and learning have been consistently observed in a number of other recent studies where rigorous diagnostic criteria for remission were applied (e.g. Van Gorp et al, 1998; Krabbendam et al, 2000; Cavanagh et al, 2002) as well as in a recent unpublished study (L. Clark, personal communication, 2002) that showed that verbal recall was still impaired following partial correlation for residual symptoms. The presence of significant impairments on executive tasks in bipolar remission has been more variable and may depend on clinical factors or the specific neuropsychological test paradigm employed. The precise functional significance of the cognitive impairment in bipolar remission needs to be examined further but may well impact on response to psychological and drug treatments. Cognitive symptoms could in fact be among the most sensitive indicators of incomplete remission.

Cavanagh, J.T. O., van Beck, M., Muir,W., et al (2002) Case-control study of neurocognitive function in euthymic patients with bipolar disorder: an association with mania. British Journal of Psychiatry, 180, 320-326.

Clark, L., Iversen, S. D. \& Goodwin, G. M. (2000) Sustained attention deficit in bipolar disorder. British Journal of Psychiatry, 180, 313-319.

Ferrier, I. N. \& Thompson, J. M. (2002) Cognitive impairment in bipolar affective disorder: implications for the bipolar diathesis. British Journal of Psychiatry, $\mathbf{1 8 0}$, 293-295.

Table I Results of partial correlation analysis on tests in which ANOVAs were significant

\begin{tabular}{llcr}
\hline & Dependent variable & Partial correlation coefficients & $P$ \\
\hline Pattern recognition memory & Proportion correct & 0.41 & 0.02 \\
Spatial recognition memory & Proportion correct & 0.31 & 0.07 \\
Delayed matching to sample & Proportion correct & 0.35 & 0.04 \\
One-touch Tower of London & Response time & -0.42 & 0.02 \\
Affective shifting task & Response time & 0.04 & 0.81 \\
\hline
\end{tabular}

Krabbendam, L., Honig, A., Wiersma, l., et al (2000) Cognitive dysfunction and white matter lesions in patients with bipolar disorder in remission. Acta Psychiatrica Scandinavica, 10I, 274-280.

Rubinsztein, J. S., Michael, A., Paykel, E. S., et al (2000) Cognitive impairment in remission in bipolar affective disorder. Psychological Medicine, 30, 1025-1036.

Van Gorp, W. G., Altshuler, L., Theberge, D. C., et al (1998) Cognitive impairments in euthymic bipolar patients with and without prior alcohol dependence. Archives of General Psychiatry, 55, 4I-45.

J. S. Rubinsztein, B. J. Sahakian Department of Psychiatry, University of Cambridge, Box 189, Addenbrooke's Hospital, Hills Road, Cambridge CB2 2QQ, UK

O'Connor et al (2002) report the effects of antenatal anxiety on behavioural/emotional problems in 4-year-old children. Their analysis of the Avon Longitudinal Study of Parents and Children (ALSPAC), a longitudinal, prospective study of women, their partners and an index child (Golding et al, 2001) takes into account a number of important covariates, including postnatal anxiety, gestational age, birth weight, and socio-economic status. They have not, however, included any measures of parenting. This is of concern because there is now a substantial body of evidence to indicate a clear association between parenting and child emotional and behavioural problems. For example, there are now a number of empirically validated models depicting the developmental progression for conduct and behaviour problems. These show a clear association between parenting practices characterised by harsh and inconsistent discipline, little positive parental involvement with the child, poor monitoring and supervision, and behaviour and conduct problems in early childhood (Patterson et al, 1989). Indeed, work using structural equation models showed that parenting and family interaction variables accounted

\section{Antenatal anxiety, parenting and behavioural/emotional problems in children}

